\title{
Pharmakologische Aspekte von mehrfach ungesättigten Fettsäuren in der parenteralen Ernährung
}

\begin{abstract}
Zusammenfassung. Trotz rasanter Entwicklung in der Intensivmedizin ist die Mortalität an Sepsis/SIRS mit 30-70\% immer noch sehr hoch. Haupttodesursache stellt das Versagen respiratorischer Funktionen bzw. anderer vitaler Organe dar. Bei der Entwicklung des Organversagens scheinen neben infektiösen (Viren, Bakterien, Pilze) auch eine Reihe nicht-infektiöser Stimuli (Gewebezerstörung, Komplementaktivierung, Immunkomplexe etc.) eine bedeutende ätiologische Rolle zu spielen. Diese entzündlichen Reaktionen haben physiologischerweise die Inaktivierung und Beseitigung des stimulierenden Agens und die Einleitung reparativer Heilungsprozesse zum Ziel. Diese natürlichen Schutzmechanismen können jedoch bei protrahierter Aktivierung humoraler und zellulärer Mediatorsysteme zu unkontrollierten Reaktionen mit Schädigung körpereigener Strukturen führen (z. B. proteolytische Zerstörung von Gewebestrukturen). Bisher fehlen jedoch suffiziente therapeutische Strategien zur Beeinflussung dieser komplexen inflammatorischen Reaktionen. Unter der Vielzahl von Entzündungsmediatoren spielen pro-inflammatorische Lipidmediatoren eine wesentliche Rolle. In zahlreichen experimentellen und klinischen Studien haben Omega-3-Fettsäuren antithrombotische und antiinflammatorische Eigenschaften bei unterschiedlichen Erkrankungen gezeigt. Diese Effekte scheinen mit der Aufnahme der in Fischöl enthaltenen Eicosapentaensäure (EPA) in die zellulären Membranphospholipidpools und deren anschließenden Metabolisierung im Zusammenhang zu stehen. Nach inflammatorischer Aktivierung wird EPA neben Arachidonsäure (AA) freigesetzt und konkurriert mit AA um die enzymatische Umsetzung durch die Zyklo- und Lipoxygenase. Die Derivate der EPA haben geringere pro-inflammatorische und chemotaktische Eigenschaften als AA-Metabolite. Im Hinblick auf prophylaktische und therapeutische Konsequenzen scheint bei der massiven Synthese von Lipidmediatoren, während inflammatorischer Reaktionen eine therapeutisch-nutritive Intervention mit Omega3-Fettsäuren sinnvoll.
\end{abstract}

Schlüsselwörter: $\omega$-3-Fettsäuren - Fischöl - Eicosapetaensäure - Lipidmediatoren - Inflammatorische Reaktion.

Pharmacological Aspects of Polyunsaturated Fatty Acids in Parenteral Nutrition. Despite immense progress in intensive-care medicine, mortality rates of $30-70 \%$ in sepsis and SIRS are still an unresolved problem. Particularly the failure of respiratory and other vital functions is a major cause of death.

\author{
A. Heller, Thea Koch \\ Institut für Anästhesiologie und Operative Intensivmedizin, \\ (Direktor: Prof. Dr. med. K. van Ackern) \\ Fakultät für Klinische Medizin Mannheim, Universität Heidelberg.
}

Besides infectious stimuli (viruses, bacteria, fungi) a variety of non-infectious triggers (tissue damage, immune complexes, complement activation, etc.) can initiate the development of organ failure. These inflammatory reactions aim physiologically towards inactivation and removal of the stimulating agents as well as the induction of reparative processes. In states of prolonged activation of humoral and cellular mediator systems the natural host defence mechanisms react in an uncontrolled manner causing tissue damage and organ failure. So far there are no efficient therapeutic strategies to influence these complex inflammatory reactions. In the development of SIRS and sepsis, pro-inflammatory lipid mediators play a crucial role. Omega-3fatty acids ( $\omega$-3-PUFAs) have shown anti-inflammatory and antithrombotic properties in a great number of experimental and clinical studies. These effects seem to be related to the uptake of eicosapentaenoic acid (EPA) into cellular membrane lipid pools and its subsequent metabolisation. After inflammatory activation EPA is released besides arachidonic acid (AA) and competes with AA for metabolisation via the cyclo- and lipoxygenase pathway. Compared to AA the derivatives of EPA have less pro-inflammatory and chemotactic characteristics. With regard to prophylactic and therapeutic consequences it appears reasonable to supplement $\omega$-3-PUFAs to attenuate the inflammatory response by modulating the generation of lipid mediators during inflammation.

Key words: Omega-3-fatty acids - Fish oil - Eicosanoids - Lipid mediators - Inflammation.

\section{Einleitung}

Die zunehmende Bedeutung von $\omega$-3-Fettsäuren ( $\omega$-3-PUFAs) geht auf epidemiologische Studien an Eskimos von Bang und Dyerberg [1] zurück. Sie konnten eine deutlich geringere Inzidenz von Thrombosen, koronarer Herzkrankheit und Myokardinfarkt bei Eskimos, die sich überwiegend von Fisch ernähren, gegenüber der Bevölkerung westlicher Industrieländer nachweisen. Im Vergleich zum europäischen Kontrollkollektiv war der Anteil von $\omega$-3-PUFAs (enthalten in Fischöl), insbesondere der mehrfach ungesättigten Fettsäuren Eicosapentaensäure (EPA) und Docosahexaensäure (DHA) in den Thrombozyten der Eskimos erhöht. Seither wurde eine Vielzahl von Studien sowohl in vitro als auch in vivo durchgeführt, die neben den benefiziellen Effekten von $\omega$-3-PUFAs auf Herz-Kreislauf-Erkrankungen [2,3], neoplastische Erkrankungen [4] und Diabetes mellitus [5] auch zeigen konnten, daß $\omega$-3-Fettsäuren antiinflammatorische und immunmodu- 
latorische Eigenschaften besitzen [6]. Diese Effekte werden mit dem vermehrten Einbau der mehrfach ungesättigten Fettsäuren EPA und DHA in den zellulären Substratpool und der Verstoffwechselung im Rahmen der Eicosanoidsynthese in Verbindung gebracht [7]. In der parenteralen Ernährung richtet sich daher ein verstärktes Interesse auf die nichtenergetischen, pharmakologischen Effekte von verschiedenen Fettsäuren.

Im Hinblick auf eine Optimierung des Ernährungsregimes stehen neben der Kettenlänge der Triglyceride vor allem das Verhältnis von $\omega-6 \mathrm{zu} \omega-3$ mehrfach ungesättigten Fettsäuren im Mittelpunkt der experimentellen und klinischen Forschung [8]. Diese essentiellen Fettsäuren, die vom Organismus nicht synthetisiert werden können und deshalb mit der Nahrung aufgenommen werden müssen, erfüllen wichtige Funktionen als Membranbausteine und Modulatoren biochemischer Prozesse. Das Fettsäuremuster der Zellmembranen ist für die Permeabilität, Elastizität und damit ihre Funktion bei Transportvorgängen und enzymatischen Reaktionen von Bedeutung. Als Vorstufen für die Synthese von biologisch hochaktiven Eikosanoiden haben die $\omega-6$ und $\omega-3$ Fettsäuren einen Einfluß auf Entzündungsreaktionen, thrombotische Prozesse, kardiovaskuläre Erkrankungen und auf die Immunabwehr [9].

Die bisher verfügbaren Daten, die auf protektive Effekte von $\omega-3$ Fettsäuren bei verschiedenen Krankheitsbildern hinweisen führten zu dem Konzept der Supplementierung der parenteralen Ernährung, mit $\omega$-3-Fettsäuren.

\section{Essentialität und Metabolismus von $\omega$-3-PUFAs}

Insgesamt ist die Menge an endogen gebildeten $\omega$-3-PUFAs im Organismus gering, und ihre Konzentration läßt sich durch exogene Zufuhr deutlich steigern. Da durch ihre strukturellen Besonderheiten $\omega$-3-Fettsäuren nicht durch $\omega$-6-Fettsäuren ersetzt werden können, treten bei langdauernder Fehlernäh- rung Mangelsymptome mit nachweisbarer Verminderung von $\omega$-3-Fettsäuren in den Lipiden von Plasma und Blutzellen [10] auf. Ein Defizit an $\omega$-3-Fettsäuren führte im Tierexperiment und beim Menschen zu neurologischen Symptomen, verminderter Sehschärfe, Wachstumsverzögerung und Hautläsionen, die sich unter Substitution von $\omega$-3-Fettsäuren zurückbildeten.

Seefisch (z.B. Hering, Lachs, Makrele, Thunfisch, Sardinen) bzw. die daraus gewonnenen Fischöle stellen die Hauptquelle für die Versorgung des Menschen mit $\omega$-3-Fettsäuren dar, da der Organismus nur in geringem Umfang in der Lage ist aus Linolensäure EPA durch Desaturation und Kettenverlängerung zu synthetisieren (Abb. 1) [11]. Omega-3-Fettsäuren wie EPA und DHA sind in Seefisch in Gewichtsanteilen von jeweils $0,1-1,2 \%$ enthalten [12]. Unter Diäten, die mit $\omega$-3-Fettsäuren angereichert waren, konnte gezeigt werden, daß sich das Verhältnis von $\omega$-3- zu $\omega$-6-Fettsäuren in der Membranzusammensetzung vieler Zellpopulationen, z.B. Erythrozyten [13], Granulozyten [14], Thrombozyten [15], Endothelzellen, Monozyten, Gehirn- [16] und Leberzellen [17] zugunsten der Pentaensäuren veränderte. In dem Konzert der dargestellten Pathomechanismen, kommt den aus der Arachidonsäure gebildeten Eicosanoiden und auch dem Plättchen aktivierenden Faktor (PAF) eine wichtige pathogenetische Bedeutung zu. So führen zahlreiche Stimuli zu einem vermehrten $\mathrm{Ca}^{2+}$ Einstrom in die Zelle und zur Aktivierung der Phospholipase $A_{2}$, die $A A$ aus dem Phospholipidpool der Membranen freisetzt (Abb.2). Da sich AA und EPA lediglich durch eine Doppelbindung unterscheiden, konkurrieren beide Fettsäuren um die Metabolisierung durch die gleichen Enzymsysteme und können sich durch ein größeres Angebot gegenseitig verdrängen [18]. In Abhängigkeit von dem Enzymmuster der jeweiligen Zelle (z.B. Thrombozyt, Endothelzelle, Leukozyt, etc.) (Abb. 3) entstehen daraus durch die Zyklooxygenase Prostaglandine und Thromboxan bzw. durch Lipoxygenase, Hydroxyfettsäuren und Leukotriene.

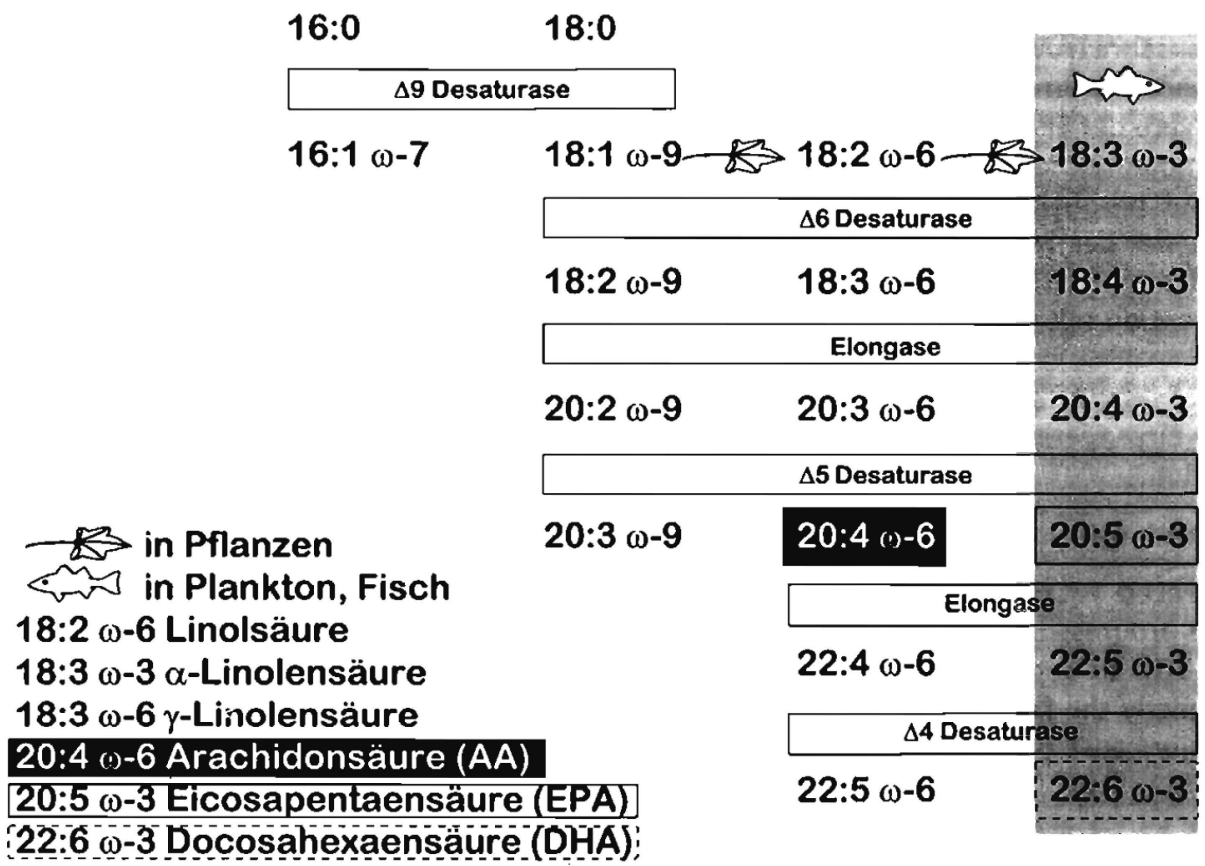

Abb. 1 Synthesewege der essentiellen Fettsäuren. Plankton und Pflanzen sind in der Lage in größerem Umfang zusätzliche Doppelbindungen in bestehende Fettsäuren einzuführen. 


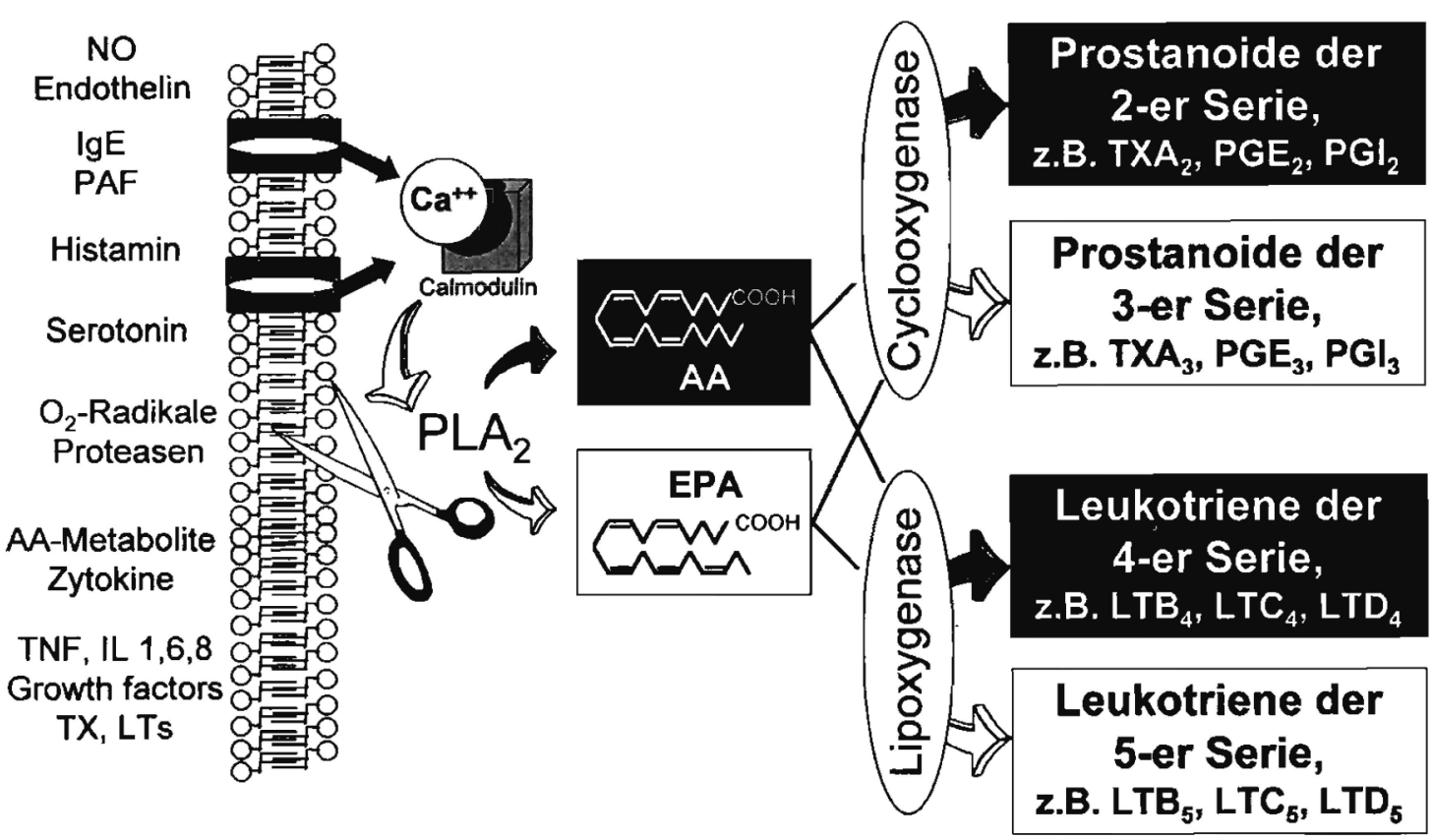

Abb. 2 Zahlreiche Stimuli führen $\mathrm{Ca}$ " vermittelt zur Aktivierung von Phospholipase $A_{2}$ $\left(P L A_{2}\right)$, die je nach Membrangehalt an $\omega$-6- oder $\omega$-3-PUFAs AA oder EPA freisetzt. Da sich $A A$ und EPA nur durch eine Doppelbindung unterscheiden, konkurrieren beide Fettsäuren um die Metabolisierung durch die gleichen Enzymsysteme.

Die aus der EPA gebildeten Derivate unterscheiden sich hinsichtlich ihrer Struktur und der biologischen Aktivität von den analogen AA-Derivaten [19]. Das von der EPA abgeleitete Zyklooxygenaseprodukt der 3er-Reihe $\mathrm{TXA}_{3}$ zeigt erheblich reduzierte proaggregatorische und vasokonstriktive Eigenschaften im Vergleich zu dem AA-abgeleiteten TXA ${ }_{2}$, während $\mathrm{PGI}_{3}$ in der antiaggregatorischen und vasodilatierenden Wirksamkeit dem $\mathrm{PGl}_{2}$ vergleichbar ist. Daher resultiert aus der Einschleusung von $\omega$-3-Fettsäuren in den Prostanoidmetabolismus ein geringerer proaggregatorischer und ein vasodilatativer Effekt. In Zellen der Granulopoese und des MonozytenMakrophagen-Systems wird AA zu den Leukotrienen der 4erSerie ( $\mathrm{LTB}_{4}, \mathrm{C}_{4}, \mathrm{D}_{4}, \mathrm{E}_{4}$ ), die potente Mediatoren der Leukozytenaktivierung, Chemotaxis und Degranulation darstellen, verstoffwechselt. EPA stellt bei der Einschleusung in den Lipoxygenaseweg sogar ein gegenüber der AA bevorzugtes Substrat der 5-Lipoxygenase dar [20]. Am Ende der enzymatischen Umsetzung entstehen die Leukotriene der 5er Serie $\left(\mathrm{LTB}_{5}, \mathrm{C}_{5}, \mathrm{D}_{5}, \mathrm{E}_{5}\right)$ mit partiell antagonistischen Eigenschaften im Vergleich zu den AA-Derivaten $[20,21]$. So besitzt LTB eine wesentlich geringere vasokonstriktorische und chemotaktische Potenz als $\mathrm{LTB}_{4}$ [19]. Auch die Bildung von PAF, der stark pro-inflammatorisch und plättchenaggregierend wirkt, wird durch EPA reduziert. Hierbei interferiert EPA ähnlich wie bei der Eicosanoidsynthese mit dem Präkursorpool von PAF [22]. Darüber hinaus ist die Inhibition der Produktion proinflammatorischer Zytokine wie Interleukin (IL)-1 und Tumor Nekrose Faktor ${ }_{\alpha}\left(\mathrm{TNF}_{\alpha}\right)$ bekannt [23]. Zur Kontrolle der Eicosanoidfreisetzung bzw. zur Blockade der entsprechenden Rezeptoren sind in den letzten Jahren eine Vielzahl von Inhibitoren entwickelt worden, die unterschiedliche Resultate auf den Verlauf verschiedener Erkrankungen zeigten [24]. Ermutigende Ergebnisse aus in vitro- und tierexperimentellen Studien konnten am Patienten teilweise nicht nachvollzogen werden. Führt man sich vor Augen, daß der Einsatz spezifischer Eicosanoidantagonisten nur die Endstrecke der Bildung einzelner Lipidmediatoren blockiert (Abb.4), erscheint der Eingriff mit $\omega$-3-PUFAs sinnvoll, um die gesamte Lipidmediatorkaskade $\mathrm{zu}$ beeinflussen ohne die physiologische Balance zwischen vasodilatierenden und vasokonstringierenden Eicosanoiden zu stören. Vor dem Hintergrund dieser biochemischen Zusammenhänge werden die Möglichkeiten einer nutritiven pharmakologischen Beeinflussung von Organfunktionen und entzündlich immunologischen Reaktionen im folgenden aufgezeigt.

\section{Bedeutung von $\omega$-3-PUFAs bei inflammatorischen Reaktionen}

Nach zahlreichen Untersuchungen kann durch exogene $\mathrm{Zu}$ fuhr von Fischöl die entzündliche Reaktion günstig beeinflußt werden [25]. Im Rahmen von inflammatorischen Reaktionen unterschiedlicher Genese kommt es zu lokaler oder systemischer Aktivierung multipler humoraler und zellulärer Mediatorsysteme. Diese inflammatorische Aktivierung des Stoffwechsels kann bei schweren Krankheitsbildern, wie Sepsis oder SIRS einen überschießenden Verlauf im Sinne einer Hyperinflammation [26] mit der Entstehung schwerer Gewebeschäden nehmen und in ein Multiorganversagen einmünden. Omega-3-Fettsäuren scheinen durch Veränderungen der Zell zu Zell Signaltransduktion in der Lage zu sein, hyperinflammatorische Prozesse abzuschwächen oder zu verhindern [27]. Dies erfolgt in der Lunge zum einen durch die beschriebene reduzierte Freisetzung von pro-inflammatorischen AA-Metaboliten und von PAF und zum anderen durch die verstärkte Bildung antiinflammatorischer EPA-Derivate, die zu einer verminderten Produktion von Zytokinen wie TNF [28], IL 1 und IL 6 [29] in Monozyten führen. Die Folge sind eine geringere Granulozyteninfiltration, eine verminderte Gefäßwandpermeabilität und eine geringere Produktion von toxischen Sauerstoffradikalen [30].

\section{Effekte von $\omega$-3 PUFAs auf die pulmonale Strombahn}

Die Lunge ist ein metabolisch hochaktives Organ das durch seine große alveoläre und kapilläre Oberfläche einer Vielzahl von endogenen und exogenen Noxen ausgesetzt ist. Daher ist die Lunge im Rahmen von Schock, Trauma und Sepsis häufig 


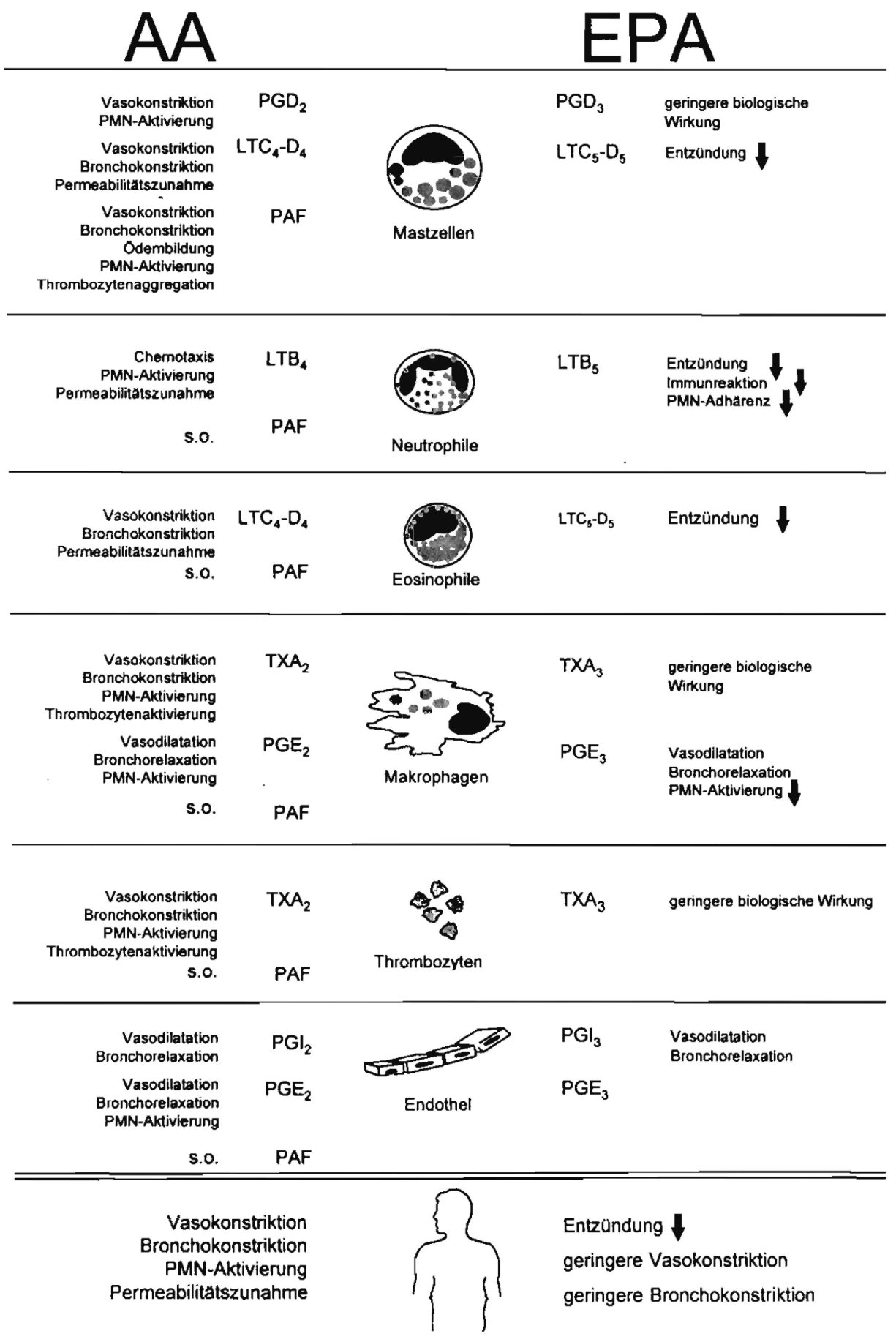

Abb.3 In Abhängigkeit von der Enzymausstattung der jeweiligen Zelle entstehen im Rahmen von inflammatorischen Vorgängen unterschiedliche Lipidmediatoren, deren Bildung durch die Applikation von mehrfach ungesättigten Fettsäuren (EPA) moduliert werden kann.

primäres Zielorgan. Bei der Entwicklung des akuten Lungenversagens, kommt es zur Aktivierung einer Vielzahl humoraler und zellulärer Mediatorsysteme, die ihrerseits zu Veränderungen des vaskulären Tonus und der Permeabilität führen (Abb. 5). Hierbei steht insbesondere $\mathrm{LTB}_{4}$ im Vordergrund, das durch seine chemotaktischen Eigenschaften zu einer erhöhten Leukozytenadhärenz am Endothel und zur Aktivierung von PMNs beiträgt. Daraus resultiert die Freisetzung von lysosomalen Proteasen und toxischen $\mathrm{O}_{2}$-Radikalen die $\mathrm{zu}$ strukturellen Schäden der alveolo-kapillären Membran und schließlich zu einem kapillären Leck führen. Klinische Befunde zeigten, daß erhöhte Thromboxan $\mathrm{A}_{2}$ - und $\mathrm{LTB}_{4}$-Spiegel im Blut und in der bronchoalveolären Lavage mit der Schwere des Lungenversagens korrelieren [31]. Vor diesem Hintergrund schien es interessant zu prüfen, ob durch die Zufuhr von $\omega$-3-PUFAs und deren Metabolisierung zu biologisch geringer aktiven Derivaten die akute inflammatorische $\mathrm{Ge}-$ fäßantwort günstig beeinflußt werden kann. In unserer Arbeitsgruppe konnten wir in einer Reihe von Untersuchungen anti-inflammatorische Wirkungen von Omega-3-Fettsäuren auf die pulmonale Strombahn zeigen. Im Hinblick auf einen möglichen therapeutischen Einsatz ergaben sich zunächst folgende Fragen, die wir in experimentellen Studien untersucht haben.

- Wie schnell werden $\omega$-3-PUFAs nach parenteraler Zufuhr in die Membranphospholipidpools eingebaut und in der pulmonalen Strombahn verstoffwechselt?

- Kommt es zu einem veränderten Eicosanoidprofil nach Fischölinfusion bei inflammatorischer Stimulation, d.h. Freisetzung von EPA aus dem zellulären Lipidpool und 


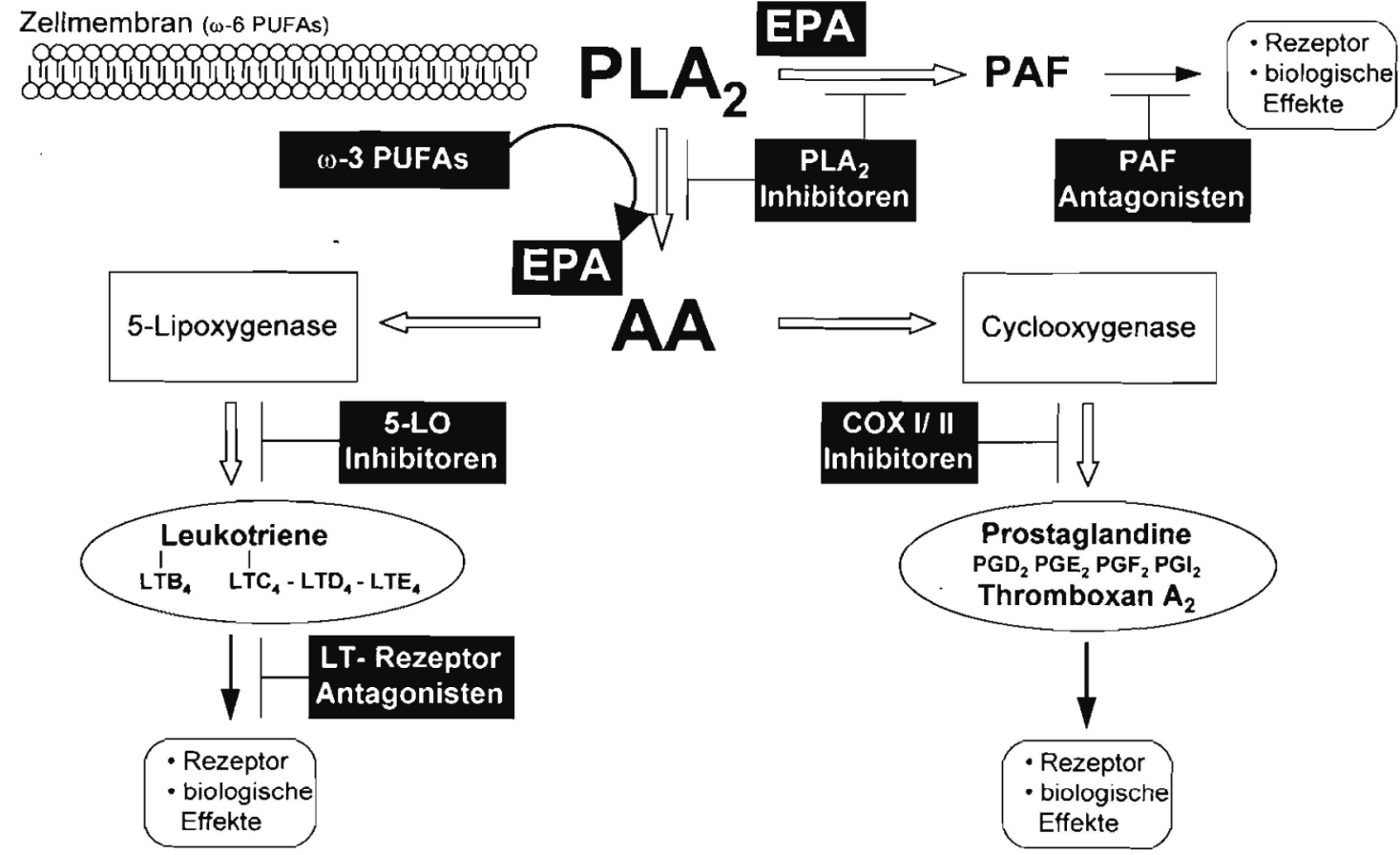

Abb. 4 Im Gegensatz zu EPA supprimieren die in den letzten Jahren entwickelten spezifischen Inhibitoren und Rezeptor-Antagonisten nur einzelne Mediatorgruppen (5-Lipoxygenase (5-LO), Zyklooxygenase $1 / \|(\operatorname{COX} 1 / 11)$, Leukotriene (L)). Phospholipase $A_{2}\left(P L A_{2}\right)$ Inhibition unterdrückt zwar die gesamte Arachidonsäurekaskade, führt aber zu Imbalancen zwischen vasodilatierenden und vasokonstringierenden Eicosanoiden [24].
Mikrobieller oder traumatischer Stimulus

Humorale Antwort:

- Komplementkaskade

- Gerinnungs- und

Fibrinolysesystem

- Kallikrein- Kininsystem
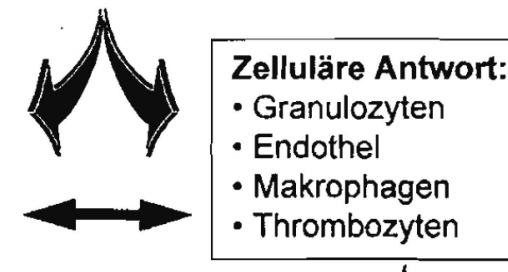

Abb.5 Pathophysiologie der Entwicklung einer mediatorinduzierten Mikrozirkulationsstörung nach mikrobiellem oder traumatischem Stimulus.

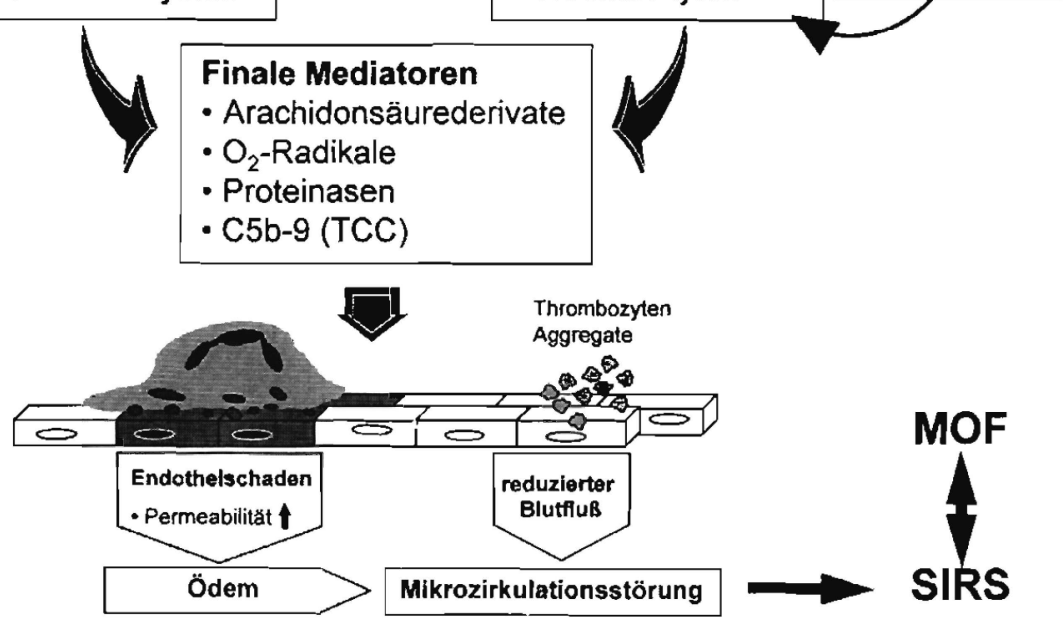

Einschleusung in den Zyklo- und Lipoxygenaseweg mit der Synthese der entsprechenden EPA abgeleiteten Derivate?

- Beeinflußt die Zufuhr von w-3-PUFAs den pulmonalen Vasotonus und die Permeabilität bei entzündlichen Krankheitsbildern im Sinne einer Protektion?

- Inwieweit wird im Hinblick auf immunologische Funktionen die bakterielle Abwehr bzw. die Lungenbesiedlung unter standardisierten Bedingungen durch $\omega$-3-PUFAs beeinflußt?

Im Gegensatz zu den zahlreichen Studien, die sich mit einer Langzeiternährung mit $\omega$-3-PUFAs über Wochen bis zu meh- reren Monaten beschäftigen [32], richtete sich unser Hauptinteresse auf die Frage, ob $\omega$-3-PUFAs schon nach kurzzeitiger parenteraler Applikation in den Phospholipidpool integriert werden und nach inflammatorischer Stimulation über ihre Metabolite einen organprotektiven Effekt ausüben. Unsere Untersuchungen wurden an isolierten und zellfrei perfundierten Kaninchenlungen durchgeführt (Abb. 6)

Zur Induktion einer unspezifischen inflammatorischen Reaktion wurde der artefizielle Stimulus $A_{23187}$-Calciumionophor gewählt, der über einen transmembranösen $\mathrm{Ca}^{2+}$-Einstrom eine Aktivierung des pulmonalen AA-Metabolismus auslöst 


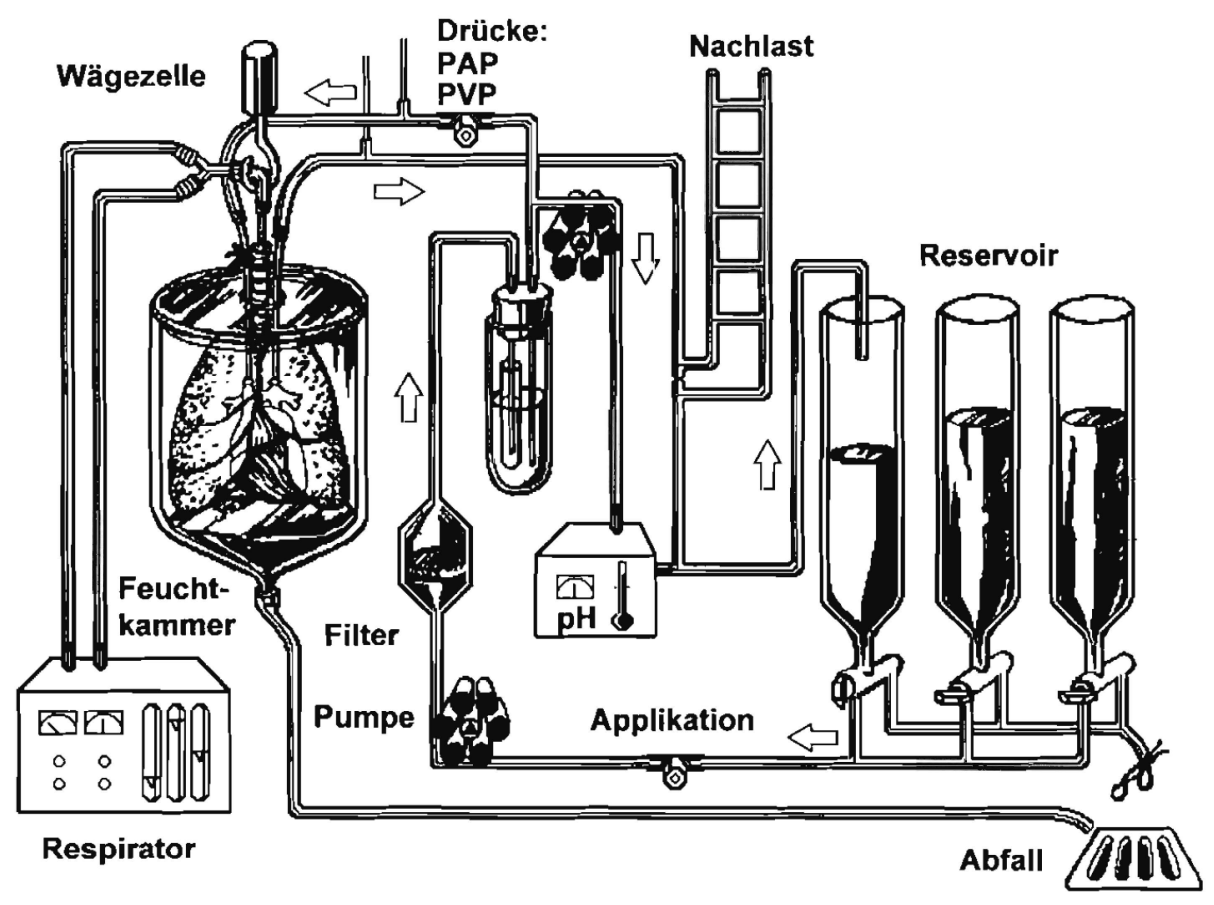

\section{Kontrollen}

sonstige Fettsăuren

Arachidonsäure

Linolsäure

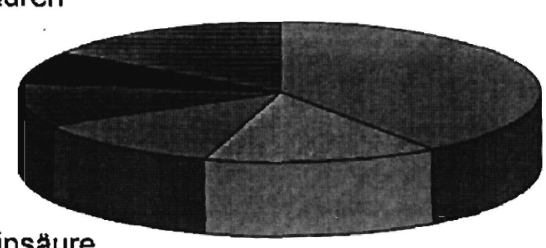

Olsäure
Abb. 6 System der isoliert perfundierten und ventilierten Kaninchenlunge. Nach Kanülierung der A. pulmonalis wurden die Lungen von anästhesierten Kaninchen isoliert und rezirkulierend mit einem Krebs-Henseleit Puffer perfundiert (Flow: $200 \mathrm{ml} / \mathrm{min}$ ). Der pulmonalarterielle Druck und die Gewichtszunahme der Lunge als Ödemindikator wurde fortlaufend registriert.
$\omega-6-P U F A s$

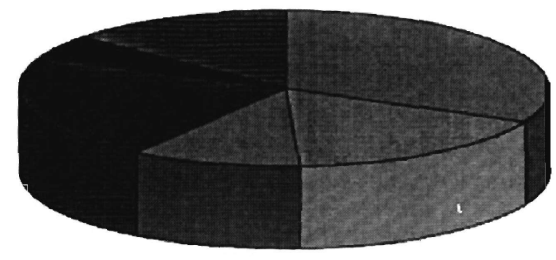

Palmitinsäure

$\omega$-3-PUFAs

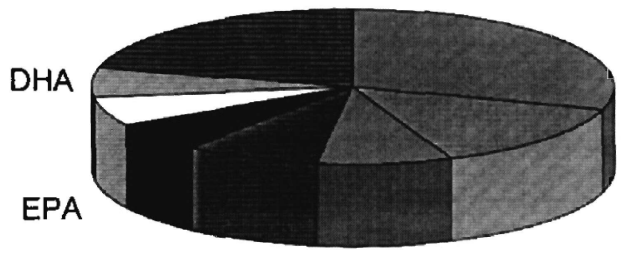

Abb. 7 Fettsäurezusammensetzung der Lungen vor (Kontrolle) und nach Perfusion mit $\omega$-3-PUFAs bzw. $\omega$-6-PUFAs. Durch die dreistündige Perfusion mit der $\omega$-3-Lipidemulsion $(0,1 \mathrm{~g} / \mathrm{kgKG})$ war es möglich den Fettsäuregehalt des Gewebes an w-3-PUFAs auf 6\% EPA und 6\% DHA anzuheben.

[33]. Schon nach dreistündiger Perfusion der Lungen mit einer Fettemulsion auf der Basis von Fischöl (Omegaven-Fresenius $\left.{ }^{(}\right)$(Tab.1) konnte ein relevanter Einbau der $\omega$-3-PUFAs EPA und DHA in die Zellmembranen nachgewiesen werden (Abb. 7). Zum Vergleich wurde Lungengewebe vor Lipidperfusion und nach Behandlung mit Sojaölemulsion (LipovenösFresenius ${ }^{\circledR}$ ) analysiert [34]. Darüberhinaus war die pulmonalvaskuläre Druckantwort auf den inflammatorischen Stimulus unter w-3-Fettsäuren deutlich abgeschwächt (Abb.8) und ging mit einer bis zu 50\% geringeren Gewichtszunahme der Lunge im Sinne einer verminderten Ödembildung einher (Abb.9) [35]. Diese Befunde konnten ebenfalls nach experimenteller Luftembolie an der isolierten Kaninchenlunge unter Perfusion mit $\omega$-3-Fettsäuren nachgewiesen werden. Die reduzierten Druckanstiege im Pulmonalkreislauf waren mit einer signifikant geringeren $\mathrm{TXB}_{2}$ Produktion verbunden [36]. Zur weiteren Abklärung der postulierten antiinflammatorischen Potenz, insbesondere der biophysikalischen Effekte der EPA-Derivate auf die Gefäßpermeabilität wurden Bestimmungen des kapillären Filtrationskoeffizienten $\left(\mathrm{K}_{\mathrm{fc}}\right)$ in der Lungenstrombahn durchgeführt. Wie erwartet stiegen die $\mathrm{K}_{\mathrm{fc}}$-Werte nach Calciumionophor-Injektion in den Kontrollpräparaten an, während die mit $\omega$-3-PUFAs vorperfundierten Lungen eine im Mittel um 50\% geringere Steigerung des $\mathrm{K}_{\mathrm{fc}}$ zeigten [37]. Diese Daten korrellierten mit einem unterschiedlichen Profil der freigesetzten Eicosanoide, die die Metabolisierung der jeweils applizierten Lipide reflektierten. Die verminderte Ödembildung unter $\omega$-3-PUFAs ging mit der Synthese EPAabgeleiteter Cysteinyl-Leukotriene einher, während die AAabgeleiteten Leukotriene und Thromboxan nur in geringen 
Tab. 1 Zusammensetzung der untersuchten Lipidemulsionen.

\begin{tabular}{|c|c|c|c|}
\hline & & $\begin{array}{l}\text { Sojaölemulsion } \\
(\mathrm{g} / \mathrm{l})\end{array}$ & $\begin{array}{l}\text { Fischölemulsion } \\
(\mathrm{g} / \mathrm{l})\end{array}$ \\
\hline Myristinsäure & C 14:0 & 0,06 & 5,39 \\
\hline Palmitinsäure & $C 26: 0$ & 12,00 & 12,12 \\
\hline Palmitoleinsäure & C $16: 1$ & - & 8,67 \\
\hline Stearinsäure & C 18:0 & 4,00 & 2,37 \\
\hline Ölsäure & C 18:1 & 22,42 & 13,57 \\
\hline Linolsäure $(\omega-6)$ & C $18: 2$ & 50,92 & 2,68 \\
\hline Linolensäure ( $\omega$-3) & C $18: 3$ & 7,21 & 0,71 \\
\hline Arachidinsäure & C 20:1 & - & 0,14 \\
\hline Arachidonsäure ( $\omega-6)$ & $C 20: 4$ & & 2,18 \\
\hline $\operatorname{EPA}(\omega-3)$ & $C 20: 5$ & & 18,88 \\
\hline Erucasäure & C $22: 1$ & & 0,71 \\
\hline DTA & C $22: 4$ & & 0,46 \\
\hline DPA & C 22:5 & & 2,52 \\
\hline $\mathrm{DHA}(\omega-3)$ & C 22:6 & & 24,58 \\
\hline Sonstige Fettsäuren & & - & 10,51 \\
\hline Glycerin & & 23,80 & \\
\hline Gesamtfettsäuren & & 106,46 & 105,40 \\
\hline
\end{tabular}

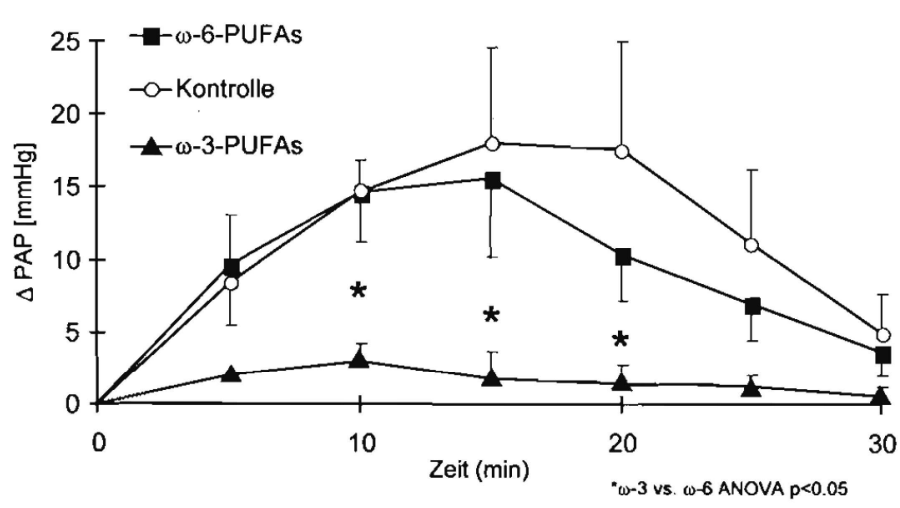

Abb. 8 Vergleich des pulmonalarteriellen Drucks in isoliert perfundierten und ventilierten Kaninchenlungen nach Applikation von $(0,1 \mathrm{~g} / \mathrm{kgKG})$ Sojaöl- oder Fischölemulsion und lipidfrei perfundierten Kontrollen.

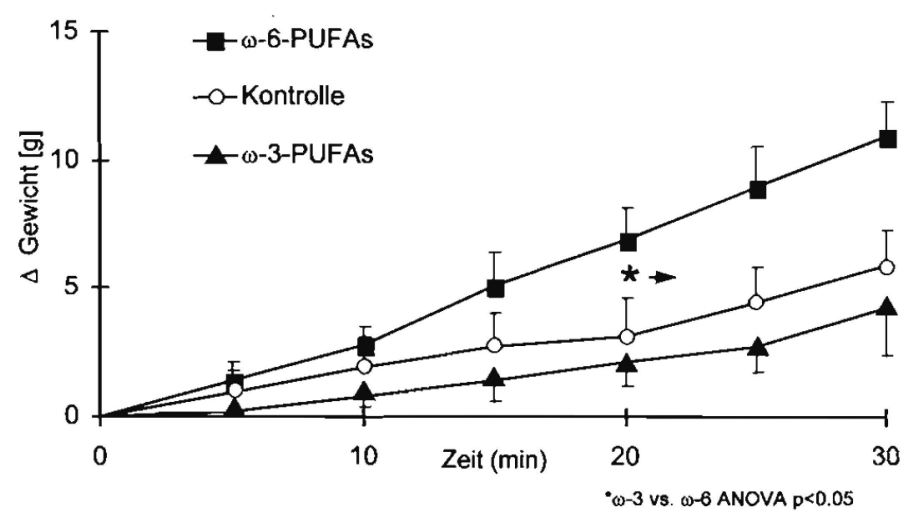

Abb.9 Vergleich der Ödementwicklung in isoliert perfundierten und ventilierten Kanichenlungen nach Applikation von $(0,1 \mathrm{~g} / \mathrm{kgKG})$ Sojaöloder Fischölemulsion und lipidfrei perfundierten Lungen.
Mengen im Perfusat nachweisbar waren (Abb.10). Die Behandlung mit $\omega$-3-PUFAs hatte jedoch im Vergleich zu den Kontroll-Lungen keinen Einfluß auf die Freisetzung des vasodilatatorischen Prostazyklins. Die Ergebnisse legen nahe, daß schon nach dreistündiger Perfusionsdauer ein Einbau von EPA ins Gewebe anzunehmen ist, die eingebaute EPA bei Aktivierung verfügbar ist und $\mathrm{zu}$ einem hinsichtlich Druck und Permeabilität günstigerem Eicosanoidspektrum führt. Weitere Studien belegen protektive Effekte auf die glatte Bronchialmuskulatur und die Schleimsekretion [38].

\section{Einfluß von $\omega$-3-PUFAs auf das Immunsystem}

Eine erhöhte Zufuhr von langkettigen $\omega$-3-PUFAs beim Menschen greift in immunologische Vorgänge ein [39]. Omega-3PUFAs beeinflussen die zellulären Abwehrfunktionen durch ihren Einbau in die Zellmembran mit einer geringeren Bildung von Prostaglandin $E_{2}$ und Leukotrien $B_{4}$ bei gleichzeitig vermehrter Synthese von Eicosanoiden mit begrenzter immunologischer Wirksamkeit wie Prostaglandin $E_{3}$ und Leukotrien $B_{5}$. Durch die verminderte Synthese des immunsuppressiv wirkenden $\mathrm{PGE}_{2}$ scheinen $\omega$-3-PUFAs im Vergleich $\mathrm{zu} \omega-6-$ PUFAs einen stimulierenden Effekt auf die zelluläre Abwehrfunktion zu entfalten. Benefizielle Effekte von $\omega$-3-PUFAs auf zelluläre Abwehrfunktionen wurden durch in vitro Untersuchungen bei kritisch kranken Patienten [40] sowie nach großen operativen Eingriffen [41] bestätigt. Im Tierexperiment konnte unter Ernährung mit $\omega$-3-Fettsäuren eine reduzierte $\mathrm{PGE}_{2}$ Produktion aus Kupferzellen gezeigt werden, die mit einer geringeren Mortalität bei Sepsis korrelierte [42]. Ferner führte die Applikation von $\omega$-3-Fettsäuren in verschiedenen in vitro und in vivo Modellen zu einer Steigerung der zellulären Immunität sowie des Opsonierungsindexes [43]. Zur Untersuchung der beschriebenen immunmodulatorischen Effekte von $\omega$-3-Fettsäuren wurde von uns ein Tiermodell etabliert, das quantitative Aussagen der Bakterien-Clearance

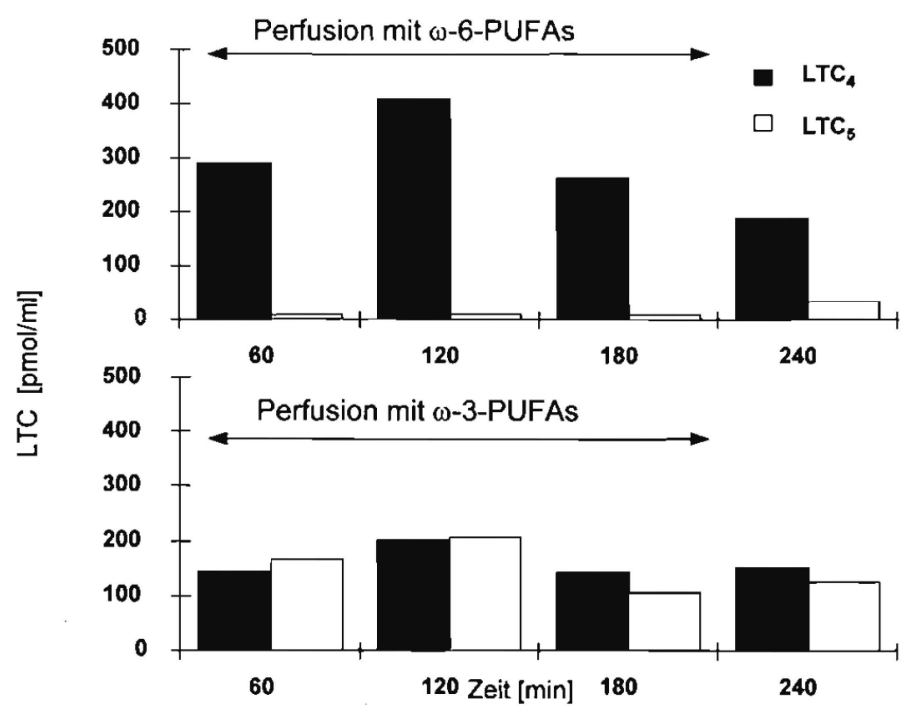

Abb. 10 Verlauf der Perfusatspiegel von Leukotrien $C_{4}$ und $C_{5}$ [pmol/ $\mathrm{ml}$ in der pulmonalen Strombahn isoliert perfundierter und ventilierter Kaninchenlungen nach Applikation von $(0,1 \mathrm{~g} / \mathrm{kgKG})$ Sojaöl- (oben) oder Fischölemulsion (unten). 
und der Organbesiedlung erlaubt [44]. Kaninchen wurden 3 Tage lang mit einer Sojaölemulsion ( $\omega-6$-PUFAs) bzw. Fischölemulsion ( $\omega$-3-PUFAs) infundiert $(0,5$ und $1,5 \mathrm{~g} / \mathrm{kg} / \mathrm{d})$ [45]. Nach der Lipidernährung erfolgte bei allen Tieren stellvertretend für die Einschwemmung von Bakterien in die Blutbahn die Injektion einer definierten Anzahl von Escherichia coli, die aus Blutkulturen eines Sepsispatienten isoliert wurden. Neben dem hämodynamischen Monitoring wurden nach E. coliInjektion in kurzen Zeitintervallen Blukulturen angelegt und nach 3 und 6 Stunden Gewebeproben von Leber, Milz, Niere und Lunge zur Beurteilung der bakteriellen Besiedlung entnommen. Abb. 11 zeigt die Bakterien-Clearance im Blut. Nach Vorbehandlung mit dem Fischölpräparat kam es zu einer signifikant schnelleren Elimination der injizierten Bakterien aus dem Blut im Vergleich zu den Sojaöl-behandelten Tieren. Während sich in Leber, Milz und Niere keine relevanten Unterschiede zeigten, ergab sich für die Lunge eine signifikant geringere Besiedlung unter der Infusion mit dem Fischölpräparat (Abb.12). Zusammenfassend zeigen die Ergebnisse unter $\omega$-3-PUFA-Infusion eine signifikant beschleunigte Bakterienclearance im Blut und eine verminderte bakterielle

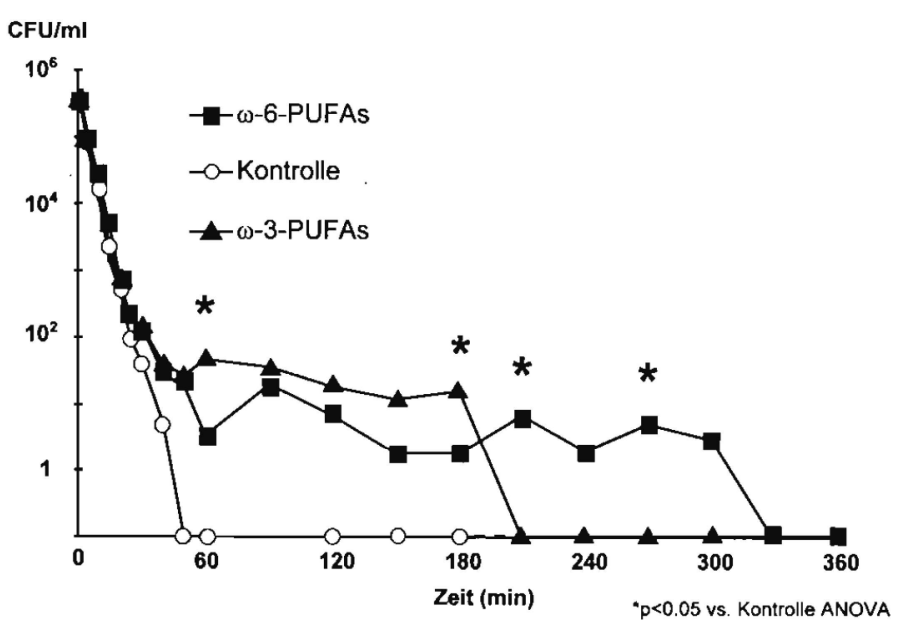

Abb.11 Bakterien-Clearance von injizierten E. coli ( $10^{7}$ colony forming units (CFU)) aus dem Blut anästhesierter Kaninchen nach Applikation von Sojaöl- oder Fischölemulsion $(0,5 \mathrm{~g} / \mathrm{kgKG} / \mathrm{d}$ über 3 Tage) bzw. $\mathrm{NaCl}$ 0,9\% (Kontrolle).

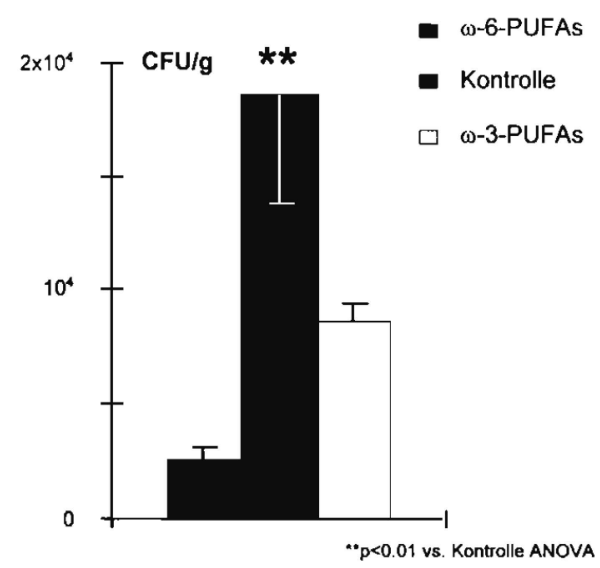

Abb. 12 Koloniezahlen von E. coli pro Gramm Gewebe in der Lunge ( $3 \mathrm{~h}$ nach Injektion von $10^{7}$ colony forming units (CFU) E.coli) anästhesierter Kaninchen nach Applikation von Sojaöl- oder Fischölemulsion ( $0,5 \mathrm{~g} / \mathrm{kgKG} / \mathrm{d}$ über 3 Tage) bzw. $\mathrm{NaCl} 0,9 \%$ (Kontrolle).
Besiedlung der Lunge. Daraus ergibt sich daß $\omega$-3-PUFAs zu einer Verbesserung der bakteriellen Abwehr insbesondere in der Lunge führen und möglicherweise zu einer verminderten Sepsisinzidenz beitragen können. In Übereinstimmung mit diesen experimentellen Befunden, die auf eine verbesserte Abwehrfunktion hinweisen, war auch in einer prospektiven klinischen Studie bei Verbrennungspatienten unter diätetischer Fischölsupplementierung eine geringere Inzidenz von Wundinfektionen, eine kürzere Behandlungsdauer und eine reduzierte Mortalität im Vergleich zu konventionellen Ernährungsregimen nachweisbar [46].

\section{Auswirkungen von $\omega-3-P U F A s$ auf die Leber}

In weiteren Studien wurden positive Effekte von Fischöl vor allem auf die Verbesserung der nutritiven Perfusion der Leber zurückgeführt [47]. Durch die Applikation von $\omega$-3-PUFAs im Endotoxinschock konnte neben einer Zunahme der intestinalen Durchblutung auch ein signifikanter Anstieg der Leberperfusion und eine Verbesserung der hepatischen Laktatclearance erzielt werden. Als Ursache für solche perfusionssteigernden Eigenschaften der $\omega$-3-PUFAs werden Verschiebungen im Eicosanoidstoffwechsel zugunsten der vasodilatierenden Metabolite der Eicosapentaensäure wie Prostaglandin $\mathrm{I}_{3}$ zusammen mit einer verstärkten endothelialen Freisetzung von Stickstoffmonoxid angesehen [48]. Ferner ergaben Studien an der Maus, daß der hepatozytäre Gehalt an Arachidonsäure, als Präkursor von vornehmlich proinflammatorisch wirksamen Mediatoren und die Prostaglandin $\mathrm{F}_{1 \alpha}$-Syntheseraten nach Fischölgabe signifikant niedriger waren als nach Verabreichung von Sojaölpräparaten [49]. Neben den Effekten einer verbesserten Perfusion wurden in mehreren tierexperimentellen Arbeiten auch direkt positive Einflüsse auf die während Sepsis inflammatorisch geschädigte Leber nachgewiesen. Als Ausdruck einer verbesserten metabolischen $\mathrm{Ge}$ samtsituation der Leber konnten Fetteinlagerungen aufgrund reduzierter Syntheseraten von Triglyceriden, Cholesterol und Phospholipiden nach Fischölzufuhr verhindert werden [50].

\section{Auswirkungen von $\omega$-3-PUFAs auf das Herz}

Kardioprotektive Effekte der $\omega$-3-PUFAs sind durch die verringerte Thrombozytenaggregation und eine verbesserte Myokardperfusion zu erklären, die aus dem veränderten Eicosanoidprofil zugunsten der vasodilatierend wirkenden Prostazyklin-Derivate resultieren. Zusätzlich können $\omega$-3-PUFAs die Wirkung des Vasodilatators NO steigern und die Sensitivität gegenüber Noradrenalin dämpfen [51]. Darüber hinaus wirken $\omega$-3-PUFAs antiarrhythmisch. Diese Effekte beruhen darauf, daß $\omega-3$ PUFAs direkt mit Ionenkanälen interagieren und zu einer reduzierten elektrischen Aktivierbarkeit von Kardiomyozyten führen. So hyperpolarisieren $\omega$-3-PUFAs das Ruhemembranpotential, erhöhen die Reizschwelle zur Auslösung eines neuen Aktionspotentials und verlängern die Refraktärzeit [52]. Parallel hierzu hemmen $\omega$-3-Fettsäuren indirekt Dihydropyridin-sensitive Kalziumkanäle des L-Typs und können eine durch Digitalis induzierte Kontraktur der Kardiomyozyten verhindern [53,54]. Omega-3-PUFAs beeinflussen weitere elektrophysiologische Prozesse, wie die Aktivität der Kalzium-Magnesium-ATPase oder von Kalium- und Natriumkanälen [55]. Neben dieser Wirkung auf transmembranöse Ionenströme reduzieren $\omega$-3-PUFAs auch die stimulierende Aktivität sympathikoadrenerger Signalübertragungssysteme. 


\section{Effekte von $\omega$-3-PUFAs bei Sepsis/SIRS}

Die Erkenntnis des Stellenwertes der Eicosanoide als wichtige Mediatoren entzündlicher Prozesse sowie die immunmodulatorische Potenz der $\omega$-3-Fettsäuren bildet die rationale Grundlage für Studien, die eine Wirksamkeit von Fischöl während Sepsis überprüfen.

In der Frühphase von Sepsis und SIRS (systemic inflammatory response syndrome) kommt es zu einer überschießenden Aktivierung humoraler und zellulärer Mediatorsysteme, die durch Veränderungen des Vasontonus und der Entwicklung eines kapillären Lecks gekennzeichnet ist und schließlich zum Organversagen führt. Aufgrund des veränderten Wirkprofils der EPA-abgeleiteten Eicosanoide wird durch die Applikation von $\omega$-3-Fettsäuren eine aniinflammatorische Intervention auf der Ebene der Lipidmediatoren möglich. Im Bereich der Leukotriene liegt die chemotaktische Aktivität und die permeabilitätsteigernde Wirkung von $\mathrm{LTB}_{5}$ um das 30-60fache niedriger als bei $\mathrm{LTB}_{4}$. Ebenso hat $\mathrm{TXA}_{3}$ ein vielfach niedrigeres proaggregatorisches und vasokonstringierendes Potential gegenüber $\mathrm{TXA}_{2}$. Während Sepsis wird vermutet, daß die reduzierte Freisetzung von $\mathrm{LTB}_{4}$ und $\mathrm{TXA}_{2}$ unter Zufuhr von $\omega$-3-Fettsäuren zu Verbesserungen in der Mikrozirkulation führt. Klinische Relevanz erlangen jene Befunde durch die Beobachtung, daß beim kritisch kranken Patienten verminderte Thromboxanspiegel und ein höheres ProstazyklinThromboxan-Verhältnis mit einer erniedrigten Mortalität korrelierten [31].

So beobachteten tierexperimentelle Studien an verschiedenen Sepsismodellen nach Zufuhr von Fischöl Verbesserungen im Energieverbrauch, der Stickstoffbilanz und Überlebensrate [56]. Verglichen mit Sojabohnenöl nahm nach Fischölzufuhr die für Sepsis charakteristische Laktatazidose als Folge einer günstigeren Sauerstoffbilanz des Gesamtorganismus ab [57].

In einer Multicenter-Studie von Bower u. Mitarb. [58] konnte eine signifikante Reduktion der mittleren Krankenhausverweildauer von im Mittel 10 Tagen bei Intensivpatienten (APACHE II Score > 10), die eine Supplementierung der enteralen Ernährung mit Fischöl (9,3 g/d), Arginin (10,4g/d) und Nukleotiden $(1,0 \mathrm{~g} / \mathrm{d})$ erhielten, im Vergleich zu einer Kontrollgruppe nachgewiesen werden. Septische Patienten schienen von der veränderten Nahrungszusammensetzung besonders zu profitieren, was sich gegenüber der Kontrollgruppe in einer Reduktion der Krankenhausverweildauer niederschlug. Darüberhinaus kam es bei allen Patienten unter FischölArginin-Nukleotid-Supplementierung zu einer Reduktion der Häufigkeit nosokomialer Infektionen. Eine kürzlich von Senkal publizierte Studie stützt diese Befunde und weist darauf hin, daß es durch die Immunonutrition zu einer Senkung der Kosten für die Behandlung postoperativer Komplikationen um 31,7\% gegenüber der konventionell behandelten Gruppe kam [59].

\section{Ausblick:}

In naher Zukunft werden zur Optimierung der parenteralen Emährung additive Fettemulsionen mit erhöhtem $\omega$-3-PUFAGehalt allgemein zur Verfügung stehen. Bei der Applikation von $\omega$-3-PUFAs scheint das Verhältnis an zugeführten $\omega-3-z u$ $\omega$-6-Fettsäuren entscheidend für die protektiven pharmakologischen Effekte eines veränderten Eicosanoidprofils zu sein [60]. Während bei den auf Sojaöl basierenden Emulsionen das $\omega-3 / \omega-6$ Verhältnis $1: 7$ beträgt, belegen eine Reihe von Studien bei einer Anreicherung der Diät mit $\omega$-3-PUFAs pharmakologische Effekte im Sinne antiinflammatorischer und geringerer proaggregatorischer und vasokonstriktiver Wirkungen. In einem Konsensuspapier der National Institutes of Health, der American Society for Parenteral and Enteral Nutrition und der American Society for Clinical Nutrition wurde der therapeutische Nutzen von $\omega$-3-Fettsäuren bei unterschiedlichen Erkrankungen dokumentiert und gefordert Forschungsaktivitäten in dieser Richtung intensiv voranzu treiben [61].

Die pharmakologischen Aspekte der $\omega$-3-PUFAs wurden in den letzten Jahren intensiv untersucht und haben zu einem neuen, differenzierten Verständnis der parenteralen Fettapplikation geführt. Da die Lipide neben ihrer Funktion als kompakte Energieträger wichtige Aufgaben als Membranbausteine und Modulatoren biochemischer Prozesse erfüllen, sollte das Ziel eines optimierten Ernährungskonzepts sein, die pharmakologischen, energetischen und essentiellen Eigenschaften der verschiedenen Fettsäuren in einer für den individuellen Patienten optimalen Weise zu kombinieren. Durch die additive Applikation von $\omega$-3-PUFAs wird eine gezielte, der Stoffwechselsituation angepaßte Substitution dieser Fettsäuren möglich. Die rasche Metabolisierung von $\omega-$ 3-Fettsäuren nach parenteraler Infusion läßt protektive Effekte innerhalb kurzer Therapiezeiten auch bei akuten Krankheitsverläufen erwarten.

\section{Literatur}

${ }^{1}$ Dyerberg J, Bang HO, Stoffersen E, Moncada S, Vane JR: Eicosapentaenoic acid and prevention of thrombosis and aterosclerosis? Lancet 1978;117-119

${ }^{2}$ Burr, ML, Fehily AM, Gilbert JF, et al.: Effects of changes in fat, fish, and fibre intakes on death and myocardial reinfarction: diet and reinfarction trial (DART). Lancet 1989;757-761

${ }^{3}$ Knapp HR, Fitzgerald GA: The antihypertensive effects of fish oil. A controlled study of polyunsaturated fatty acid supplements in essential hypertension. N. Engl. J. Med. 1989;320:1037-1043

${ }^{4}$ Jenski LJ, Zerouga M, Stillwell W: Omega-3 fatty acid containing liposomes in cancer therapy. Proc. Soc. Exp. Biol. Med. 1995;210: $227-233$

${ }^{5}$ Westervelt HT, de Graaf JC, van Breugel HH, Akkermann JW, Sixma JJ, Erkelens DW, Banga JD: Effects of low-dose EPA-E on glycemic control, lipid profile, lipoprotein (a), platelet aggregation, viscosity, and platelet and vessel wall interaction in NIDDM. Diabet. Care 1993;16:683-688

${ }^{6}$ Burton JL: Dietary fatty acids and inflammatory skin disease. Lancet 1989;27-30

${ }^{7}$ Weber PC: Fish oil fatty acids and cardiovascular function: epidemiology and biochemical mechanisms. Biochem. Soc. Trans. 1990;18:1045-1049 
${ }^{8}$ Heller A, Koch T: Omega-3-Fettsäuren als adjuvante Therapie bei inflammatorischen Reaktionen. Anästhesiol. Intensivmed. 1996; 10 (37):517-528

${ }^{9}$ Endres S, DeCaterina R, Schmidt EB, Kristensen SD: n-3 Polyunsaturated fatty acids: update 1995. Eur. J. Clin. Invest. 1995; 25:629-638

${ }^{10}$ Connor WE, Neuringer M, Reisbick S: Essentiality of $\omega 3$ fatty acids: Evidence from the primate model and implications for human nutrition. In: Simopoulos AP, Kifer RR, Martin RE, Barlow SM (eds.): Health effects of $\omega 3$ polyunsaturated fatty acids in seafoods. World Rev. Nutr. Diet. Karger, Basel 1991;66:118-132

11 Diboune M, Ferard G, Ingenbleek Y, Bourguignat A, Spielmann D, Scheppler-Roupert C, Tulasne PA, Calon B, Hasselmann M. Sauder P: Soybean oil, blackcurrant seed oil, medium-chain triglycerides, and plasma phospholipid fatty acids of stressed patients. Nutrition 1993; 9(4):344-349

${ }_{12}$ Simopoulos AP, Kifer RR, Martin RE (Hrsg.): Health effects of polyunsaturated fatty acids in seafood. Academic Press, Orlando, Florida 1986

${ }^{13}$ Brown AJ, Pang E, Roberts DCK: Persistent changes in the fatty acid composition of erythrocyte membranes after moderate intake of n-3 polyunsaturated fatty acids: study design implications. Am. J. Clin. Nutr. 1991;54:668-673

${ }^{14}$ Chilton $\mathrm{FH}$, Patel $\mathrm{M}$, Fonteh AN, Hubbard WC, Triggiani M: Dietary n-3 fatty acid effects on neutrophil lipid composition and mediator production. Influence of duration and dosage. J. Clin. Invest. 1993;91:115-122

${ }^{15}$ Croset M, Bayon Y, Lagarde M: Incorporation and turnover of eicosapentaenoic and docosahexaenoic acids in human blood platelets in vitro. Biochem. J. 1992;281:309-316

16 Tocher DR, Bell JG, Sargent JR: Incorporation of $\left({ }^{3} \mathrm{H}\right)$ arachidonic and $\left({ }^{14} \mathrm{C}\right)$ eicosapentaenoic acids into glycerophospholipids and their metabolism via lipoxygenases in isolated brain cells from rainbow trout oncorhynchus myciss. J. Neurochem. 1991;6: $2078-2085$

${ }^{17}$ Bourre JM, Bonneil M, Dumont O, Piciotti M, Calaf R, Portugal H, Nalbone $\mathrm{G}$, Lafont $\mathrm{H}$ : Effect of increasing amounts of dietary fish oil on brain and liver fatty acid composition. Biochim. Biophys. Acta 1990;1043:149-152

${ }^{18}$ Strasser T, Fischer S, Weber PC: Leukotriene B is formed in human neutrophils after dietary eicosapentaenoic acid. Proc. Natl. Acad. Sci. USA 1985;82:1540-1543

${ }^{19}$ Lee TH, Sethi T, Crea AEG, Peters W, Arm JP, Horton CE, Walport MJ, Spur W: Characterization of leukotriene $B_{3}$ : comparison of its biological activities with leukotriene $B_{4}$ and leukotriene $B_{5}$ in complement receptor enhancement, lysozyme release and chemotaxis of human neutrophils. Clin. Sci. 1988;74:467-475

${ }^{20}$ Lee TH, Hoover RL, Williams JD, Sperling RI, Ravalese J, Spur BW, Robinson DR, Corey EJ, Lewis RA, Austen KF: Effect of dietary enrichment with eicosapentaenoic and docosahexaenoic acids on in vitro neutrophil and monocyte leukotriene generation and neutrophil function. N. Engl. J. Med. 1985;312:1217-1224

${ }^{21}$ Lewis RA. Lee TH, Austen KF: Effects of omega-3 fatty acids on the generation of products of the 5-lipoxygenase pathway, In: Simopoulos AP (Hrsg.): Health effects of polyunsaturated fatty acids in seafoods. Academic Press, Orlando 1986;227-238

${ }^{22}$ Sperling RI, Robin J-L, Kylander KA, Lee TH, Lewis RA, Austen KF: The effects of $n-3$ polyunsaturated fatty acids on the generation of platelet-activating factor-acether by human monocytes. J. Immunol. 1987; 139:4186-4191

${ }^{23}$ Cooper A, Gibbons L, Horan M, Little R, Rothwell N: Effect of dietary fish oil supplementation on fever and cytokine production in human volunteers. Clin. Nutr. 1993;12:321-328

${ }^{24}$ Heller A, Koch T, Schmeck J, van Ackern K: Lipid mediators in inflammatory disorders. Drugs 1998; in press
${ }^{25}$ Kinsella J, Lokesh B, Broughton S, Whelan J: Dietary polyunsaturated fatty acids and eicosanoids: Potential effects on the modulation of inflammatory and immune cells. An overview. Nutrition 1990;6:24-43

${ }^{26}$ Faist E, Hartl WH, Baue AE: Immunmechanistik der posttraumatischen Hyperinflammation und Sepsis. Immun. Infekt. 1994; 22(6):203-213

${ }^{27}$ Grimm H, Tibell A, Norrlind B, Schott J, Bohle RM, Papavassilis C, Führer D, Mayer K, Grimminger F: Immunsuppressivität parenteraler Fettemulsionen bei definierter Immunstimulation. Infus. Ther. Transfus. Med. 1995;22:110-116

${ }^{28}$ Chandrasekar B, Fernandes G: Decreased pro-inflammatory cytokines and increased antioxidant enzyme gene expression by omega-3 lipids in murine lupus nephritis. Biochem. Biophys. Res. Commun. 1994;200(2):893-898

${ }^{29}$ Molvig J, Pociot F, Worsaae H, Wogensen LD, Baek L, Christensen P. Mandrup Poulsen T, Andersen K, Madsen P, Dyerberg J: Dietary supplementation with omega-3-polyunsaturated fatty acids decreases mononuclear cell proliferation and interleukin- 1 beta content but not monokine secretion in healthy and insulindependent diabetic individuals. Scand. J. Immunol. 1991;34(4): $399-410$

${ }^{30}$ Foeg M, Thomas G, Ramwell P: Free radicals, arachidonic acid metabolism, and nutrition. J. Parenteral and Enteral Nutr. 1990;5 (Suppl. 14):218-222

31 Oettinger W, Peskar B, Begar H: Profiles of endogenous prostaglandin $F_{2 \alpha}$, thromboxane $A_{2}$ and prostacyclin with regard to cardiovascular and organ functions in early septic shock in man. Eur. Surg. Res. 1987;19:65-67

${ }^{32}$ De Catarina R, Giannessi D, Mazzone A, Bernini W, Lazzerini G Maffei S, Cerri M, Salvatore L, Weksler B: Vascular prostacyclin is increased in patients ingesting $\mathrm{n}-3$ polyunsaturated fatty acids prior to coronary bypass surgery. Circulation 1990;82:428-438

${ }^{33}$ Seeger W, Walmrath D, Grimminger F, Rosseau S, Schütte $H$, Krämer H-J, Ermert L, Kiss L: Adult Respiratory Distress Syndome: Model systems using isolated perfused rabbit lungs. Meth. Enzym. 1994;233:549-584

${ }^{34}$ Koch T, Duncker H-P, Klein A, Schlotzer E, Peskar BM, van Ackern $K$, Neuhof $H$ : Modulation of pulmonary vascular resistance and edema formation by short term infusion of a $10 \%$ fish oil emulsion. Infusions Ther. Transfus. Medizin 1993;20:291 - 300

${ }^{35}$ Koch T, Duncker HP, Klein A, Neuhof H, van Ackern K: Effects of omega-3-fatty acids on pulmonary vascular reaction: A therapeutical approach in critical illness. Clin. Intens. Care 1993;4 (Suppl. 2): 10

${ }^{36}$ Koch T, Heller A, Schlotzer E, Neuhof H: Effekte einer nutritiven anti-inflammatorischen Intervention mit Omega-3 Fettsäuren auf die pulmonale Strombahn. Intensivmed. 1997;34, Suppl. 1: 43

${ }^{37}$ Koch T, Heller A, Breil I, van Ackern K, Neuhof H: Alterations of pulmonary capillary filtration and leukotriene synthesis due to infusion of a lipid emulsion enriched with Omega-3-fatty acids. Clin. Intens. Care 1995;6:112-120

${ }^{38}$ Lawrence R, Sorrell T: Eicosapentaenoic acid in cystic fibrosis: evidence of a pathogenic role for leukotriene $B_{4}$. Lancet 1993;342:465-469

${ }^{39}$ Suchner U, Senftleben U: Immune modulation by polyunsaturated fatty acids during nutritional therapy: Interactions with synthesis and effects of eicosanoids. Infusionther. Transfusionsmed. 1994;21:167-182

${ }^{40}$ Alexander JW: Augmentation of host defense reactivity with special nutrients. In: Faist E, Meakins JL, Schildberg FW (eds.): Host defense dysfunction in trauma, schock and sepsis. Springer, Heidelberg 1993, pp 995-1001

${ }^{41}$ Lieberman MD, Shou J, Torres AS, et al.: Effects of nutrient substrate on immune function. Nutrition 1990;6:88-91 
${ }^{42}$ Barton RG, Wells CL, et al.: Dietary omega-3 fatty acids decrease mortality and Kupffer cell prostaglandin $E_{2}$ production in a rat model of chronic sepsis. J. Trauma 1991;31:768-773

${ }^{43}$ Hinds A, Sander TAB: The effect of increasing levels of dietary fish oil rich in eicosapentaenoic and docosahexaenoic acid on lymphocyte phospholipid fatty acid composition and cellmediated immunity in the mouse. Br. Nutr. 1993;69:423-429

${ }^{44}$ Koch T, Heller S, van Ackern K, Schiefer HG, Neuhof H: Impairment of bacterial clearance induced by norepinephrine in rabbits. Intensive Care Med. 1996;22:637-643

${ }^{45}$ Breil I, Koch T, Prechtl A, Heller A, Schlotzer E, Schiefer G, van Ackern $\mathrm{K}$, Neuhof $\mathrm{H}$ : Effects of lipid emulsions containing polyunsaturated fatty acids (omega-3 versus omega-6) on bacterial clearance. Crit. Care Med. 1996; 24(11):1893-1902

${ }^{46}$ Alexander JW, Gottschlich MM: Nutritional immunomodulation in burn patients. Crit. Care Med. 1990;18:149-153

${ }^{47}$ Pscheidl E, Wan J, Blackbum G, Bistrian B, Istfan N: Influence of $\omega-3$ fatty acids on splanchnic blood flow and lactate metabolism in an endotoxemic rat model. Metabolism 1992;41:698-705

${ }^{48}$ Vanhoutte PM: Fish oil and endothelium-dependent relaxations. In: II. International Conference on the Health Effects of Omega-3 Polyunsaturated Fatty acids in Seafoods 1990

${ }^{49}$ Lokesh B, Licari J, Kinsella J: Effect of different dietary triglycerides on liver fatty acids and prostaglandin synthesis by mouse peritoneal cells. J. Parent. Enteral. Nutr. 1992;16:316-321

50 Yeh SL, Chen WJ, Huang PC: Effects of fish oil and safflower oil emulsions on diet-induced hepatic steatosis in rats receiving total parenteral nutrition. Clin. Nutr. 1996;15:80 - 83

${ }^{51}$ Schini VB, Durante W, Catovsky S, Vanhoutte PM: Eicosapentaenoic acid potentiates the production of nitric oxide by interleukin 1-beta in cultured vascular smooth muscle cells. J. Vasc. Res. 1993;30:209-217

52 Kang JX, Xiao YF, Leaf A: Free, long-chain, polyunsaturated fatty acids reduce membrane electrical excitability in neonatal rat cardiac myocytes. Proc. Natl. Acad. Sci. USA 1995;92:3997-4001

${ }^{53}$ Hallaq H, Sellmayer A, Smith TW, Leaf A: Protective effects of eicosapentaenoic acid on oubain toxicity in neonatal rat cardiac myocytes. Proc. Natl. Acad. Sci. USA 1990;87:7834-7838

${ }^{54}$ Hallaq H, Smith TW, Leaf A: Modulation of dihydropyridinesensitive calcium channels in heart cells by fish oil fatty acids. Proc. Natl. Acads. Sci. USA 1992;89:1760-1764

55 Kinoshita I, Itoh K, Nishida-Nakai M, Hirota H, Otsuji S, Shibata $\mathrm{N}$ : Antiarrhythmic effects of eicosapentaenoic acid during myocardial infarction: enhanced cardiac micosomal $\left(\mathrm{Ca}^{2+}\right.$ $\mathrm{Mg}^{2+}$ )-ATPase activity. Jpn. Circ. J. 1994;58:903-912

${ }^{56}$ Johnson JA III, Griswold JA, Muakkassa FF: Essential fatty acids influence survival in sepsis. J. Trauma 1993;35(1):128-131

${ }^{57}$ Pomposelli J, Flores E, Lopes S, Blackburn G, Zeisel S, Bistrian B: Diets enriched with $n-3$ fatty acids ameliorate lactic acidosis by improving endotoxin-induced tissue hypoperfusion in guinea pigs. Ann. Surg. 1991;213:166-176

58 Bower RH, Cerra FB, Bershadsky B, Licari JJ, Hoyt DB, Jensen GL, van Buren CT, Rothkopf MM, Daly JM, Adelsberg BR: Early enteral administration of a formula (Impact ${ }^{\circledR}$ ) supplemented with arginine, nucleotides, and fish oil in intensive care unit patients: Results of a multicenter, prospective, randomized, clinical trial. Crit. Care Med. 1995;23(3):436 - 449

${ }^{59}$ Senkal M, Mumme A, Eickhoff U, Geier B, Spath G, Wulfert D, Joosten U, Frei A, Kemen M: Early postoperative enteral immunonutrition: Clinical outcome and cost comparison analysis in surgical patients. Crit. Care Med. 1997;25(9):1489 - 1496

${ }^{60}$ Boudreau M, Chanmugam P, Hart S, Lee S, Hwang D: Lack of dose response by dietary $\omega$-3-fatty acids at a constant ratio of $\omega-3$ to $\omega$-6-fatty acids in suppressing eicosanoid biosynthesis from arachidonic acid. Am. J. Clin. Nutr. 1991;54:111 - 117
${ }^{61}$ Klein S, Kinney J, Jeejeebhoy K, Alpers D, Hellerstein M, Murray M, Twomey P: Nutrition support in clinical practice: Review of published data and recommendations for future research directions. JPEN 1997;21:133-156

\section{PD Dr. Thea Koch}

Institut für Anästhesiologie und Operative Intensivmedizin Fakultät für Klinische Medizin Mannheim Theodor-Kutzer-Ufer 1 - 3 68167 Mannheim E-mail: heller@rumms.uni-mannheim.de 